\title{
A methodology for control structure adaptation in presence of varying, unknown sub-system interaction degree
}

\author{
Anca MAXIM* ${ }^{* \ddagger}$, Dana COPOT ${ }^{*}$, Clara IONESCU* and Robin DE KEYSER* \\ ${ }^{*}$ Ghent University, Research group on Dynamical Systems and Control \\ Technologiepark 914, 9052 Ghent, Belgium \\ Email: claramihaela.ionescu@ugent.be \\ ‡’Gheorghe Asachi' Technical University of Iasi, Department of Automatic Control and Applied Informatics \\ Blvd. Mangeron 27, 700050 Iasi, Romania
}

\begin{abstract}
This paper proposes a rationale and methodology for control structure adaptation in presence of varying, unknown sub-system interaction degree. Two elements are introduced: 1) the detection, i.e., the cycle of detecting changing circumstances, planning and deploying responsive modifications and 2) the adaptation of the control architecture to maintain specified performance, fulfilled in absence of model information. The first hand results presented in this work indicate the method works well. Simulation studies support the application potential of the proposed methodology.
\end{abstract}

\section{INTRODUCTION}

Plant-wide control implies to some extent information exchange along several layers in both horizontal and vertical directions of the overall's system architecture [1]. The problem of control architecture adaptation to model changes has gained renewed interest from the community. Factory of the future is a concept involving multiple sub-systems, which operate individually, but interact with each other in the framework of plant-wide control and operation.

Driven by the continual quest for enhancing performance and robustness, machines, production cells and their components evolved from simple devices to complex cyber-physical systems, i.e., networks of interacting physical and computational components [2]. The current industrial control approach is inadequate to cope with the complex, often uncertain and/or varying dynamic behaviour of these systems, leading to suboptimally performing systems, unreliable behaviour and even instability.

Large scale systems, which have to deal with increasing raw material and energy costs, tighter operating conditions, stringent safety and ecological specifications have become highly integrated modern plants [3]. These plants consist of a multitude of sub-systems, which execute different tasks and where the interaction (either from task execution or task failure) propagate in the entire network through direct and indirect loops [3], [4].

A mathematical foundation for analysis of sub-system interaction exists and provides a clear view on the various classes of interaction one may have in such large scale plants [4]. The classification is made on the following features: i) direct or indirect and combinations on these, based on time and route of interaction. Plant-wide operability is obtained based on unit interactions between sub-systems and dissipativity of each individual unit [3]. These units can be homogeneous or heterogeneous. The connection of these units is also of great importance, e.g. sequential or parallel.

Mostly, the interactions are unknown and assumptions are made upon. These assumptions are usually formulated on bounding values which reflect stability of the overall system. Adaptation of the plant-wide control architecture is revisited when a task or target of a sub-system cannot be met, i.e., due to constraints or due to interaction, which changes from weak to strong [5].

Decentralized adaptive control for uncertain interconnected systems is a well known topic of research in the community and a vast amount of literature reports various solutions [6], [7], [8]. Some equivalence principle and conservative assumptions have led to manifold of works but did not penetrate successfully the industrial landscape, mainly due to inability to satisfy overall stability and performance of the controlled network. This has been changed significantly when backstepping has been introduced in the 1990s. Recent works provide interesting solutions for overall convergence in the presence of nonlinear dynamics and unknown interactions [8]. The interactions introduce complexity in loop dynamics and simple or multivariable PID controllers may prove unable to deal with them, leading to poor performance. As a result, nonPID controllers for complex multivariable processes have been developed for industrial applications [6], [7].

In this paper we propose a simple yet effective methodology for control structure adaptation of interconnected sub-systems, by means performance monitoring and use of PID auto-tuning methods to obtain new controller parameters. The assumptions are formulated such that realistic conditions are emulated. The procedure is tested in simulation mimicking a sequential process with interaction representative for process industry. The paper is structured as follows. In Section II, the proposed methodology is formulated and the PID auto-tuning algorithm is described. In Section III the results are presented and discussed. The conclusions are provided in Section IV. 


\section{Proposed Methodology}

\section{A. Scenario}

The current industrial control approach for these interconnected systems is often a decentralized PID-like control approach, that focuses on controlling each sub-system separately. Consequently, the achievable robustness and performance is limited, and can no longer meet the continuously increasing economic (installation and operation costs vs. productivity/profit), and ecology demands. The current academic state-of-the-art in control design is much more advanced than the current industrial control practice. Control design methodologies that can cope with complex system dynamics and uncertainty are available [9], [10], [11], [12], [13]. Despite their great potential, these design methodologies have yet to make their way to industrial applications. This is mainly because they typically result in complex controllers and expert knowledge is required to use them.

Hereafter, we propose the following scenario and approach for the control structure adaptation in the presence of interconnection between various sub-systems. Figure 1 illustrates the concept as a dynamic scenario divided into three phases, corresponding to the three steps in the methodology summarized below. Consider two sub-systems $S_{1}^{0}$ and $S_{2}^{0}$, for which an individual PID controller exists $\left(P I D_{1}^{0}\right.$ and $P I D_{2}^{0}$, respectively) and works according to the given specifications. In this case the sub-systems have weak interaction, which can be dealt with using decentralized control. This controller is designed via any procedure, but industrial applications commonly employ CAD tools for tuning the respective $K_{p}, T_{i}$ and $T_{d}$ parameter values, or automatic PID tuners [15]. This decentralized closed loop scheme runs from time instant $t_{0}$ until time instant $t_{1}$.

Consider also a performance index to monitor the closed loop performance [14]. For instance, in each loop $i, i=1,2$, the absolute error can be used as a performance index $J_{i}$, where 0 represents ideal performance (i.e., output follows the setpoint value):

$$
J_{i}=\left|e_{i}(t)\right|
$$

with $e_{i}(t)=r_{i}(t)-y_{i}(t)$, where $r_{i}$ and $y_{i}, i=1,2$ denote the desired reference trajectory and the measured output, respectively. It is noteworthy to mention that in the presence of noisy disturbances, the error signal must be first filtered, and then used to compute the index. For simplicity, in figure 1 only the evolution of $J_{1}$ is depicted.

At the time instant $t_{1}$, the interaction between the subsystems changes from weak to strong. The coupling effects, which are propagated between the two decentralized control loops disturb the performance, and the index deteriorates increasing above 0 . If interaction is very strong, it can lead to de-stabilizing effects. In order to avoid this drastic effect, a tolerance limit for the performance index may be introduced based on the knowledge of the system response in the initial phase of the experiment. For example, an operator-defined value established during the nominal running of the process can be used. In practice, when setting the tolerance limit, one needs to take into account effects of noise.

Following the values of the performance index, if these remain below the tolerance limit, then no additional action is required. However, if the index evolves beyond these limits, then the control architecture must be adapted to take into account this new situation and improve the overall closed loop performance.

\section{B. Auto-tuning procedure}

At this point we introduce the assumption that the loops remain stable at all times, which is also valid for the autotuning algorithm result. As soon as the performance index degrades, multivariable iterative PID tuning rules, as given in [15] can be executed. The tuning can be re-executed to maintain performance in presence of varying dynamics of MIMO loops. At time instant $t_{2}$, the control architecture is adapted (with new values for PID parameters, obtained in the auto-tuning phase) and the performance index is restabilized towards its zero values. Note that in this work the auto-tuning procedure starts only when unexpected interaction effects decrease the closed loop performance [16]. Setpoint changes are not considered in the performance evaluation because in process control applications these occur at higher time intervals. Note that the procedure can also be applied for ramp setpoints, usually found in mechatronic industrial processes, but this paper is more focused towards process control applications. The convergence of the algorithm depends on the time constants of the process and the number of iterations is factorial dependent with the input-output pairs.

Briefly, the auto-tuning procedure can be summarized in the following steps: 1) close one loop with a relay feedback and leave open the other loop; 2) in the first loop, replace the relay with the proportional controller; 3 ) add a delay after the relay block in the closed loop of the second output while maintaining the proportional controller on the other loop; 4) compute a PID controller for the second loop based on the MM algorithm from [17]; 5) replace the relay+delay from the loop selected at step 3 with the PID from Step 4;6) add a delay after the relay block in the closed loop of the first output; 7) compute a PID controller for the first loop using the MM algorithm; 8) repeat steps 5-7. The convergence of the algorithm is established when the output magnitude and phase values in the relay+delay test vary less than $5 \%$ from those obtained in the relay test. A detailed description of the auto-tuning procedure is given in [15].

\section{ILLUSTRATIVE EXAMPLE}

In this paper, we address all dynamical systems represented as a first order plus dead time (FOPDT) transfer function:

$$
P(s)=\frac{K}{T s+1} e^{-L}
$$

with $s$ the Laplace operator, $T$ the time constant, $L$ the time delay, and relative delay given by:

$$
\tau=\frac{L}{T+L}
$$




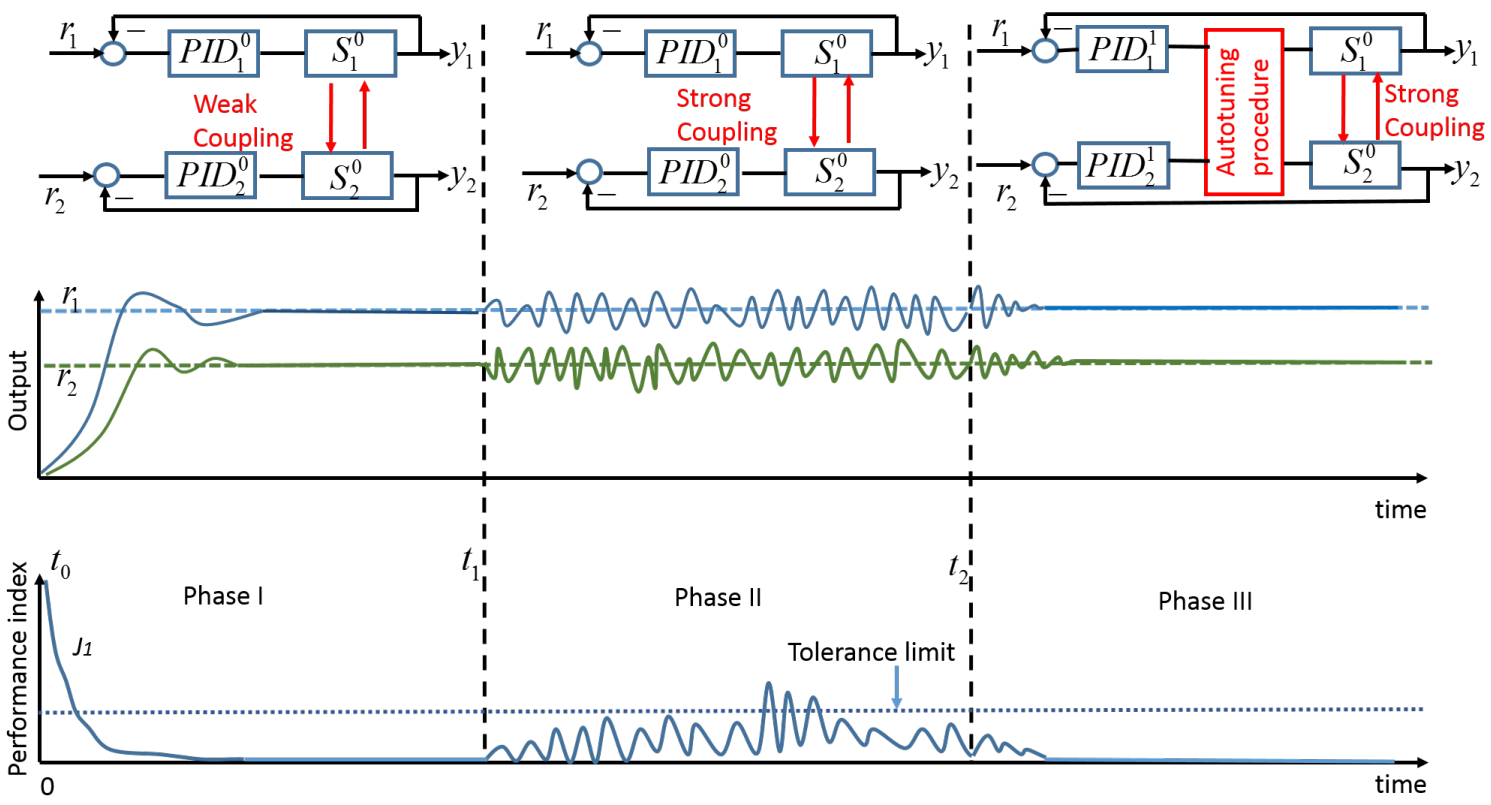

Fig. 1. Conceptual view of the three dynamic phases of the process with sub-system interaction and control architecture adaptation.

further considered lag dominant for $\tau<0.5$ and delay dominant for $\tau>0.5$. The $2 \times 2$ process matrix transfer function is then considered to be generically defined as:

$$
G(s)=\left[\begin{array}{cc}
P_{11}(s) & k 2 \cdot P_{12}(s) \\
k 3 \cdot P_{21}(s) & P_{22}(s)
\end{array}\right]
$$

with $k 2, k 3$ gains of interaction between the loops and $P(s)$ are based on the process defined in (2), with unit gain and variations in the delay values $L$. A system with low interaction was defined for values of $k 2, k 3<0.3$ and with high interaction for values of $k 2, k 3>0.5$.

As an illustrative example, the case of a quadruple water tank system as in the Quanser benchmark (www.Quanser.com), described in [15] is used. The

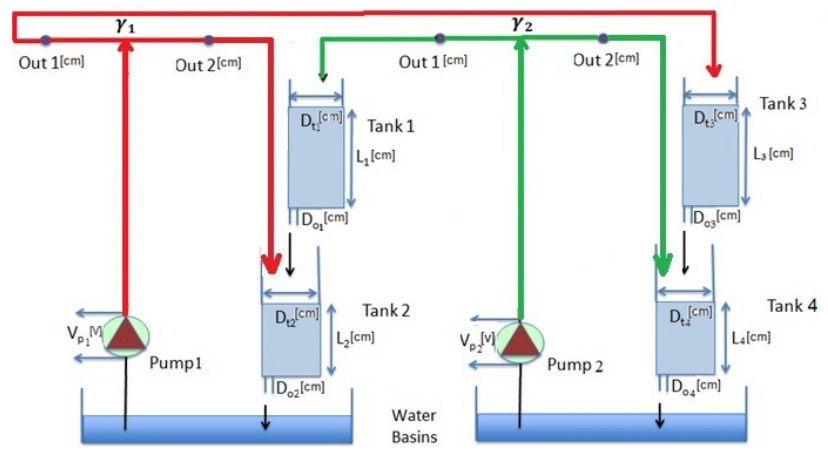

Fig. 2. Schematic representations of the quadruple tank process

schematic representation of the process is depicted in figure 2 , with the model given in [15]:

$$
G(s)=\left[\begin{array}{cc}
\frac{2.48}{168.2 s^{2}+25.94 s+1} & \frac{1.417}{16.02 s+1} \\
\frac{1.283}{14.59 s+1} & \frac{2.917}{219.1 s+29.61 s+1}
\end{array}\right]
$$

Firstly, the $2^{\text {nd }}$ order transfer functions from (5) are approximated with two FOPTD.

The performed experiment according with the conceptual description from figure 1 is the following:

- Phase I - weak interaction between the sub-systems $\left(k_{2}=k_{3}=0.1\right)$ is assumed.

- the transfer function matrix (5) becomes:

$$
\tilde{G}_{\mathrm{LAG}}(s)=\left[\begin{array}{cc}
\frac{2.48}{20 s+1} e^{-7 s} & \frac{0.28}{16.02 s+1} \\
\frac{0.25}{14.59 s+1} & \frac{2.91}{23 s+1} e^{-8 s}
\end{array}\right]
$$

Following the iteration procedure in II.B:

-the parameters for $P I D_{1}^{0}$ are: $K_{p_{1}}=0.48, T_{i_{1}}=8.33$, $T_{d_{1}}=2.08$.

-the parameters for $P I D_{2}^{0}$ are: $K_{p_{2}}=0.42, T_{i_{2}}=9.52$, $T_{d_{2}}=2.38$.

- Phase II - strong interaction between the sub-systems $\left(k_{2}=k_{3}=0.6\right)$ is introduced, while the controllers remain the same $\left(P I D_{1}^{0}, P I D_{2}^{0}\right)$.

- the transfer function matrix (6) becomes:

$$
\tilde{G}_{\mathrm{LAG}}(s)=\left[\begin{array}{cc}
\frac{2.48}{20 s+1} e^{-7 s} & \frac{0.98}{16.02 s+1} \\
\frac{0.89}{14.59 s+1} & \frac{2.91}{23 s+1} e^{-8 s}
\end{array}\right]
$$

- the process controllers remain the same as in Phase I.

- Phase III - strong interaction between the sub-systems $\left(k_{2}=k_{3}=0.6\right)$ is assumed, but the architecture is changed and different controllers $\left(P I D_{1}^{1}, P I D_{2}^{1}\right)$ obtained via auto-tuning are employed.

-for the process with strong interactions (7), the autotuning procedure begins once the performance index reaches an imposed threshold.

-the new parameters for $P I D_{1}^{1}$ are: $K_{p_{1}}=0.44, T_{i_{1}}=$ 


\section{$2.09, T_{d_{1}}=2.27$.}

-the new parameters for $P I D_{2}^{1}$ are: $K_{p_{2}}=0.38, T_{i_{2}}=$ $10.52, T_{d_{2}}=2.63$.

In phase I of the experiment, when the sub-systems have low interaction, the PID controllers were tuned with a robustness specification of $50 \%$. In phase III, a specification of $60 \%$ was demanded in the auto-tuning procedure.

Figures 3(a) and 3(b) depict the closed loop results (and corresponding performance index) for the entire experiment, namely: i) first phase from time instant $0 s$ to $600 \mathrm{~s}$, ii) second phase starting at time instant $600 \mathrm{~s}$ when the interaction changes from weak to strong by changing the gains $k_{2}$ and $k_{3}$ from 0.1 to 0.6 , respectively and iii) the final phase beginning at time instant $687 \mathrm{~s}$ corresponding with the new control architecture (that is, with the new controllers obtained after the auto-tuning procedure is finalized).
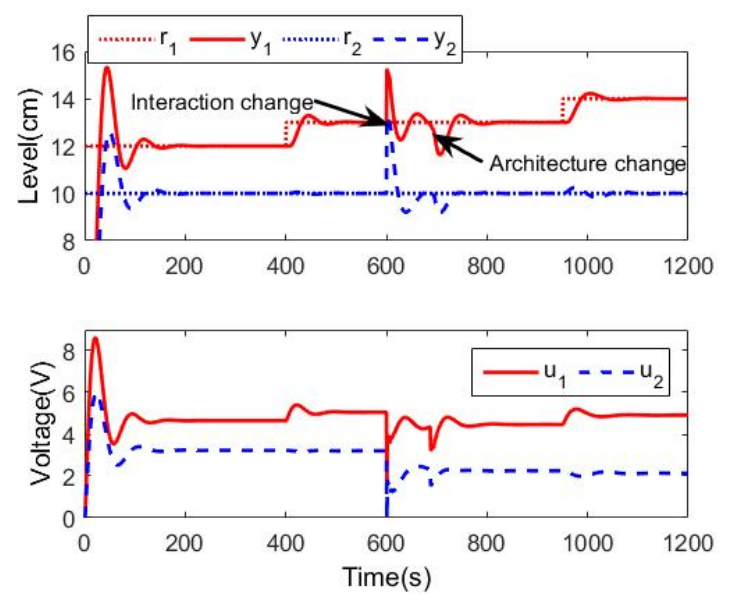

(a) Reference tracking results, where the outputs $y_{1}$ and $y_{2}$ correspond with the water levels $L_{2}$ and $L_{4}$, respectively.

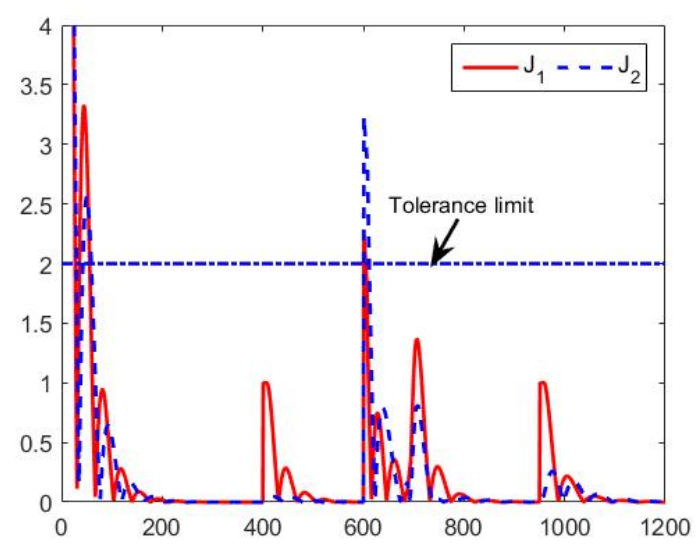

(b) Absolute error index $J_{i}$, where $i=1,2$ corresponds with the loop number

Fig. 3. Closed loop performance in lag dominant experiment

The simulated results from figure 3(a) and 3(b) suggest that during first phase, while the interaction is weak, the absolute error performance indexes remain below the tolerance limit. In our case, the limit value 2 for the absolute error suffices. In phase two, the performance indexes crossing of the imposed tolerance limit is recorded and the auto-tuning procedure is started up. At the time instant $687 \mathrm{~s}$ a new control architecture is adopted, and the system returns to its initial performance.

\section{CONCLUSION}

In this paper a novel control methodology, which deals with interacting sub-systems is presented. The concept uses a performance index (i.e., the absolute error) to monitor the system evolution and to change the control architecture (by means of PID auto-tuning) when the performance deteriorates beyond a given tolerance interval. Although only simulation results are provided, the methodology can be easily adapted for a more realistic experiment in the presence of noise and/or disturbances.

Further developments aim to investigate the limitations of the current methodology in such conditions.

\section{REFERENCES}

[1] G.P. Rangaiah (Editor), V. Kariwala (Editor), Plantwide control: Recent Developements and Applications, Wiley, 2012.

[2] I. Horváth, Beyond advanced mechatronics: new challenges of socialcyber-physical systems, Proceedings of the 1st Workshop on Mechatronic Design, Linz, Austria, 2012.

[3] R. Setiawan, J. Bao, Analysis of interaction effects on plantwide operability, Ind Eng Chem Res, 50(14), 8585-8602, 2011

[4] M.L. Climent, Analysis of subsystem interactions, Kybernetes, 32(9/10), 1504-1514, 2003

[5] H.M. Kim, N.F. Michelena, P. Y. Papalambros, T. Jiang, Target cascading in optimal system design, Transactions of the ASME, 125, 474-480, 2003

[6] Q-G Wang,Y. Zhang, M-S Chiu, Non-interacting control design for multivariable industrial processes, Journal of Process Control, 13(3), 253265,2003

[7] C. Wang, Y. Lin, Decentralized adaptive tracking control for a class of interconnected nonlinear time-varying systems, Automatica, 54(-), 16-24, 2015

[8] L. Liu, X-J Xie, Decentralized adaptive stabilization for interconnected systems with dynamic input-output and nonlinear interactions, Automatica, 46(6), 1060-1067, 2010

[9] L. Magni, R. Scattolini, Stabilizing decentralized model predictive control of nonlinear systems, Automatica, 42(7), 1231-1236, 2006.

[10] W.J. Cai, W. Ni, M.J. He, C.Y. Ni, Normalized Decoupling-A New Approach for MIMO Process Control System Design, Ind. Eng. Chem. Res., 47(19), 7347-6356, 2008.

[11] S. Di Cairano, A. Bemporad, I.V. Kolmanovsky, D. Hrovat, Model predictive control of magnetically actuated mass spring dampers for automotive applications, Int. J. of Control, 80(11), 1701-1716, 2007.

[12] A.H. Richards, J.P. How, Robust distributed model predictive control, International Journal of Control, 80(9), 1517-1531, 2007.

[13] P. Christofides, R. Scattolini, D. Munoz de la Pena, J. Liu, Distributed model predictive control: A tutorial review and future research directions, Computers and Chemical Engineering, 51(-), 21-41, 2013.

[14] Z.V. Rekasius, A general performance index for analytical design of control systems, IRE Trans on Autom Ctrl, 6(2), 217-222, 1961

[15] C.M. Ionescu, A. Maxim, C. Copot, R. De Keyser, Robust PID Autotuning for the quadruple tank system, Proc. of the IFAC Symp on Dynamics and Control of Process Systems including Biosystems (DYCOPS), 2016, IFAC NMO, 919-924, DOI:10.1016/j.ifacol.2016.07.313, Trondheim, Norway

[16] C-C. Yu, Autotuning of PID Controllers: A relay feedback approach, Springer, 2nd edition, 2006.

[17] R. De Keyser, O. Joita, C. Ionescu, The next generation of relay-based pid autotuners (part 2): A simple relay based PID autotuner with specified modulus margin. In IFAC Conf. on Advances in PID Control, Brescia, Italy, 6 pages, 2012. 